\title{
AIP
}

\section{Bias effects on the electronic spectrum of a molecular bridge}

Heidi Phillips, Alexander Prociuk, and Barry D. Dunietz

Citation: J. Chem. Phys. 134, 054708 (2011); doi: 10.1063/1.3531695

View online: http://dx.doi.org/10.1063/1.3531695

View Table of Contents: http://jcp.aip.org/resource/1/JCPSA6/v134/i5

Published by the AIP Publishing LLC.

\section{Additional information on J. Chem. Phys.}

Journal Homepage: http://jcp.aip.org/

Journal Information: http://jcp.aip.org/about/about_the_journal

Top downloads: http://jcp.aip.org/features/most_downloaded

Information for Authors: http://jcp.aip.org/authors

\section{ADVERTISEMENT}

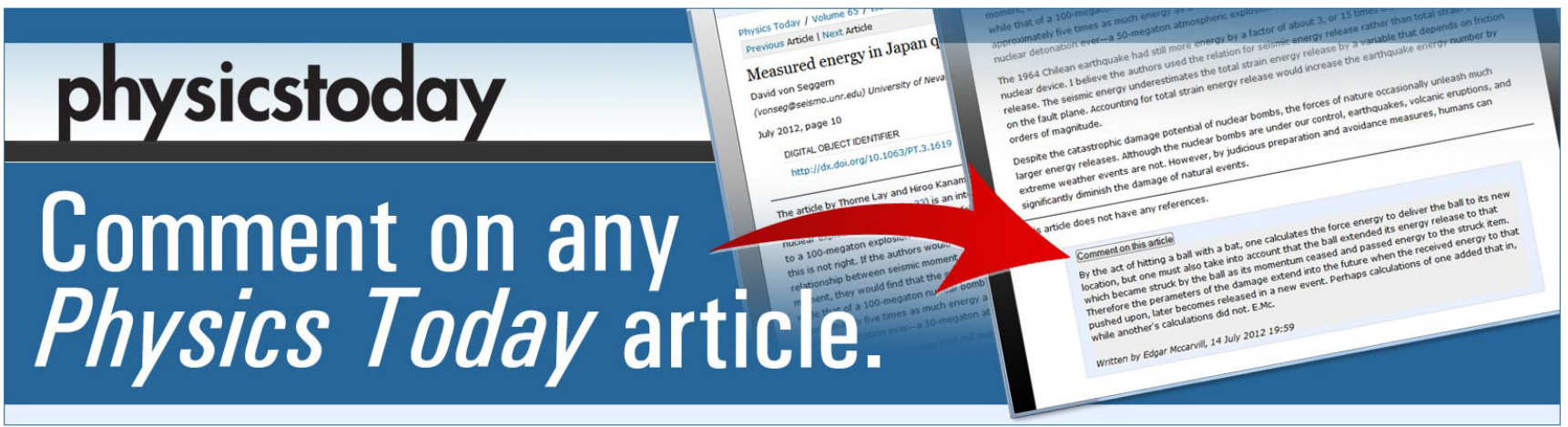




\title{
Bias effects on the electronic spectrum of a molecular bridge
}

\author{
Heidi Phillips, Alexander Prociuk, and Barry D. Dunietz ${ }^{\mathrm{a})}$ \\ Department of Chemistry, University of Michigan, Ann Arbor, Michigan 48109, USA
}

(Received 23 June 2010; accepted 6 December 2010; published online 7 February 2011)

\begin{abstract}
In this paper the effect of bias and geometric symmetry breaking on the electronic spectrum of a model molecular system is studied. Geometric symmetry breaking can either enhance the dissipative effect of the bias, where spectral peaks are disabled, or enable new excitations that are absent under zero bias conditions. The spectral analysis is performed on a simple model system by solving for the electronic response to an instantaneously impulsive perturbation in the dipole approximation. The dynamical response is extracted from the electronic equations of motion as expressed by the Keldysh formalism. This expression provides for the accurate treatment of the electronic structure of a bulk-coupled system at the chosen model Hamiltonian electronic structure level. (C) 2011 American Institute of Physics. [doi:10.1063/1.3531695]
\end{abstract}

\section{INTRODUCTION}

Advances in bulk-bridging by nanoscale and molecular scale wire fabrication motivate researchers to explore the function of these systems as electron transporting devices. A wide range of experimental schemes ${ }^{1-12}$ are used to fabricate molecular transport junctions, where typically large statistical sampling and elaborate control schemes are required. It therefore remains a challenge to identify a robust, controllable, and reproducible molecular junction fabrication scheme. Computational transport modeling through molecular systems has the potential to address this difficulty and has indeed demonstrated strong conductance dependence on geometric aspects related to the bonding at the interface. ${ }^{13,14}$ Proper characterization of bulk-coupled molecular systems is crucial for establishing a meaningful comparison between modeled and measured conductance.

Another promising prospect for enhancing characterization of molecular junctions under bias is offered by combining spectroscopy and current measurements. The charging of a small molecular bridge during the conductance process is coupled with changes in nuclear configuration. These geometric changes underlie the strong dependence of electron transport (ET) on vibrational degrees of freedom. Experimentally, the vibrational spectra of single molecules are obtained by inelastic electron tunneling spectroscopy (IETS). ${ }^{15-17}$ This procedure has been accompanied by substantial progress in modeling phonon-assisted conduction. ${ }^{18-22}$ Other experimental developments combine optical spectroscopy with transport studies, ${ }^{23-27}$ whereby conductance enhancement of the junction is correlated with changes of the in situ measured Raman spectra.

The proper modeling of the optical response of biased systems requires a time-dependent (TD) treatment. Such treatments have been recently extended to consider dynamical aspects of electron transport, where the current under transient conditions and driven by TD biasing conditions was resolved. ${ }^{28-37}$ We model the spectrum which involves calculating the junction's electronic density response to an instan-

\footnotetext{
a)Electronic mail: bdunietz@umich.edu.
}

taneous impulse in time. The dynamical response is obtained by solving the electronic equations of motion (e.o.m.s) that are expressed using the Keldysh formalism. A TD first order perturbation theory (PT) level (linear response) is used to simplify the expressions. This approach is appropriate to fundamentally model the electronic electrode-coupled system. In Secs. II and III we describe the model and method used. In Sections IV and V we discuss the results and finish with conclusions. Our analysis is performed on a simple model system of a pair of carbon atoms coupled to two gold wires, which allows us to access basic electronic spectral dependence on the applied bias.

In this study, bias-induced nonequilibrium effects on the electronic spectra of electrode-coupled systems are analyzed at the fundamental level. We illustrate the basic effect of bias on the spectrum of a molecular bridge. The electronic density of states (DOS) of the bridge is broadened due to the projection of the electrodes' DOS on the bridge. The bulk coupling, therefore, also broadens the spectral peak, as illustrated in Fig. 1 by comparing 1 (a) to 1(b). We then demonstrate the qualitative effect of the bias on the electronic transitions. The electronic transitions between the molecular bands are greatly affected by the bias. ${ }^{35}$ The applied bias leads to dynamical flux through the broadened energy levels. The dynamical electron flux disables excitations between states that differ in their occupation at the equilibrium state and which support the current under bias. Likewise, the bias enables excitations between transporting states that are equally populated at equilibrium. See Fig. 1(c) for illustration of these effects of the bias on the spectra. In the following, we quantify the disabling and enabling effect of the bias on a model molecular level. We also correlate the bias and symmetry breaking modes of the molecular bridge to the electronic spectrum. We consider different coupling strengths between bridge and the source and between bridge and the drain electrodes [see Fig. 1(d)].

\section{MODEL}

The electronic spectra of biased molecular junctions are studied using the model system as illustrated in Fig. 2. The simple model consists of a pair of one-dimensional wires as 


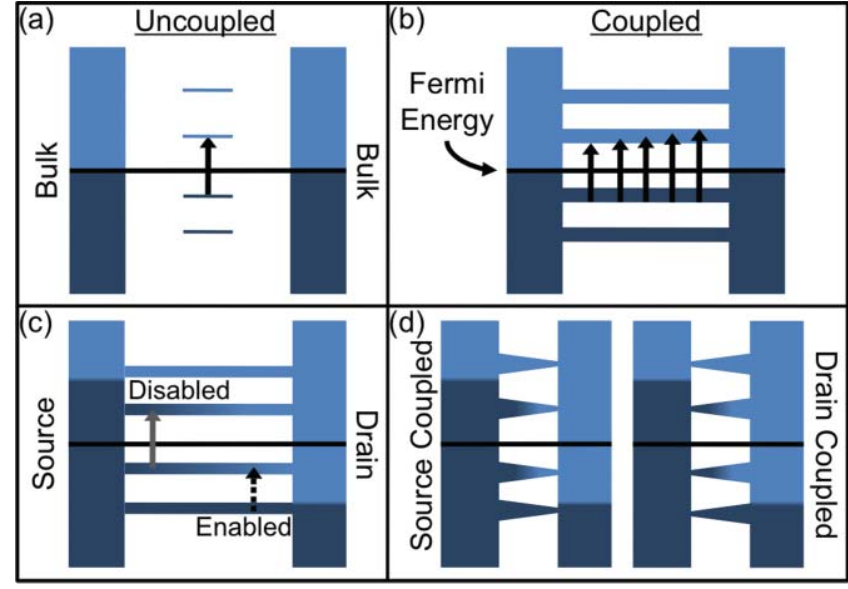

FIG. 1. The effect of coupling to electrodes and bias is illustrated. (a) The electronic spectra of the uncoupled molecule are resolved (only HOMOLUMO transition is implied). (b) The spectral peak of the molecular bridge is broadened due to the coupling to electrodes. (c) The bias may enable or disable electronic transitions. (d) Mechanical symmetry breaking may lead to stronger coupling to the source or drain electrode.

the electrodes and two sites oriented perpendicularly to the wires as the junction. Each site is described using a single s-type Gaussian basis function, which is spherically isotropic relative to the center of the site. The spherical function size is set to the electronic radius of a gold atom (for wire sites) or carbon atom (for the junction sites).

We then identify a "core" region consisting of the two carbon atoms and the two center gold atoms between which the carbons are situated. The gold atoms within the wires are spaced $2.88 \AA$ apart and the two core gold atoms are spaced $1.90 \AA$ apart. The carbon bond length is set to $3.29 \AA$. In a previous study we found that this "diamond like" structure at the simple model Hamiltonian level provides an opportunity to probe the electronic spectrum of an electrode-coupled system affected by an applied bias. ${ }^{35}$

Specifically, the atomic orbital Hamiltonian matrix is parameterized based on the ionization potential and evaluated using the following Huckel-type expressions:

$$
\begin{aligned}
& H_{A, A}=-I_{A}, \\
& H_{A, B}=-\frac{1}{2} K\left(I_{A}+I_{B}\right) S_{A, B},
\end{aligned}
$$

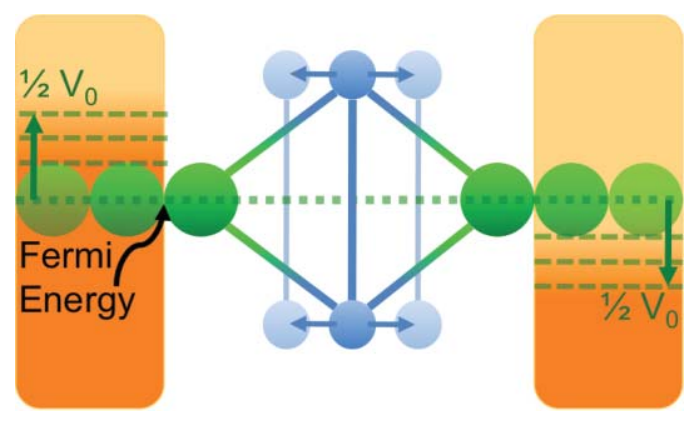

FIG. 2. Schematic diagram representing junction region. Effect of potential bias is implied as well as the shift of carbons toward source or drain electrode.
TABLE I. Radii, ionization potentials, $I_{A}$, and Gaussian basis set coefficients $\alpha$ for $\mathrm{C}$ and $\mathrm{Au}$.

\begin{tabular}{lccc}
\hline \hline Atom & Radius $(\AA)$ & $I_{A}(\mathrm{eV})$ & $\alpha\left(\AA^{-2}\right)$ \\
\hline $\mathrm{C}$ & 0.77 & 11.26 & 1.074 \\
$\mathrm{Au}$ & 1.44 & 9.22 & 0.307 \\
\hline \hline
\end{tabular}

where $S_{A, B}$ is the atomic orbital overlap between the s-type basis functions centered on atoms $A$ and $B, I_{A}$ is the ionization potential for atom $A$, and $K$ is a constant set to 1.75. The numerical values for these parameters are provided in Table I. The Hamiltonian is then orthogonalized $\left(\mathbf{H} \rightarrow \mathbf{S}^{-1 / 2} \mathbf{H S}^{-1 / 2}\right)$ followed by the imposition of a tightbinding condition within the electrode regions, where only on-site and nearest-neighbor hopping elements are nonzero. All site and hopping elements are maintained in the four-site region of the orthogonalized Hamiltonian that corresponds to the $\mathrm{Au}-\mathrm{C}_{2}-\mathrm{Au}$ region of the preorthogonalized Hamiltonian. In addition, this Hamiltonian is padded with electrode wires of nine gold atoms on each side of the perpendicular $\mathrm{C}_{2}$ system to ensure that edge effects are minimized in the orthogonalization procedure.

We obtain the electronic spectrum from the dynamic electronic density of the system upon response to relevant perturbations. As described in the following, the inherent twotime (time correlation) properties of the Keldysh propagators can be used to formally extract the evolving electronic energy distribution density $\rho(E, t)$, thereby providing access to the responding electronic density $\rho(t)$ of the perturbed, electrodecoupled junction:

$$
\rho(t)=\int d E \rho(E, t)
$$

In this representation, the energy distribution reflects the band structure of the junction coupled with the electrodes. The energy bands have finite widths that support current through scattering states that couple both electrodes. For example, a DOS peak centered at $E^{\prime}$ with a $\Delta E$ width describes the effectively infinite number of states resulting from the projection. In general, $\rho(E, t) d E$ contributes states to the density matrix $(\rho(t))$ with energies in the range $E$ to $E+d E$.

In the following, we study the effects of geometric symmetry breaking (by nuclear configuration shifts) of voltage biased systems on their electronic spectra. The steady potential bias is represented by chemical potential shifts from the Fermi energy with an opposite sign on each of the electrodes, as shown in Fig. 2. The repulsive (attractive) charges of the electrons (holes) in the left (right) electrode induce a ramping bias across the bridge. We then obtain the electronic density response to an impulsive pulse following the dipole approximation (i.e., the pulse is $\mathcal{E}_{0} \delta(t) \hat{D}$, where $\hat{D}$ is the dipole operator) to generate the time-dependence of the dipole moment,

$$
\langle D(t)\rangle=\int d E \operatorname{Tr}[\rho(\mathrm{E}, \mathrm{t}) \hat{\mathrm{D}}] .
$$

The temporal response of the dipole moment $(D(t))$ contains the excitation spectrum of the biased system, where peaks 

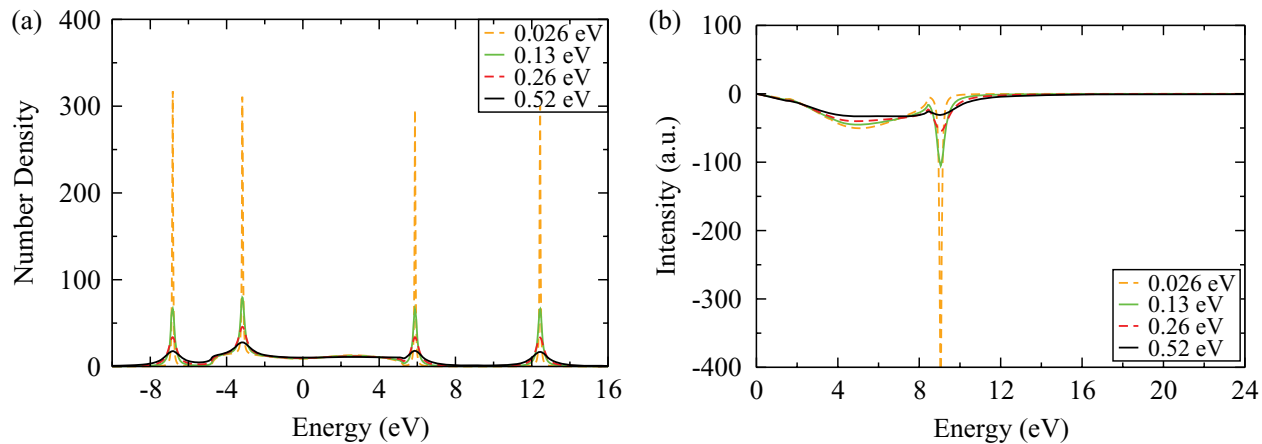

FIG. 3. (a) The electronic DOS under increasing broadening factor. (b) The electronic spectra corresponding to increasing broadening factor-

in the frequency representation $\left[\tilde{D}(\Delta \omega)=\int e^{i \Delta \omega t} D(t) d t\right]$ correspond to the excitation energies.

The electronic DOS projected on the bridging atomic region at equilibrium (zero applied bias) are shown in Figs. 3(a) and 4(a). As discussed further below, in the first figure we consider the electronic spectrum affected by electrode-induced electronic DOS broadening, and in the second figure we consider geometrically induced symmetry breaking effects. The electronic spectrum is calculated using the dipole moment oriented along the $\mathrm{C}-\mathrm{C}$ bond ( $y$-axis). In all calculations, the energy scale is shifted to set the Fermi energy to zero for convenience.

\section{METHOD}

Next, we describe our approach for evaluating the evolving electronic density that is used to derive the electronic spectrum of a molecular junction under bias conditions. We solve the electronic e.o.m.s using Keldysh formalism. In accordance with previous reports, we introduce the following change in notation: the energy distribution variable $E$ is replaced by $\bar{\omega}$, and the time variable $t$ by $\bar{t}^{33}$

In Keldysh formalism, electron dynamics is represented by the lesser Green's function (GF),

$$
G^{<}\left(x t, x^{\prime} t^{\prime}\right) \equiv i \frac{\operatorname{Tr}\left[\hat{U}\left(t_{0}-i \beta, t_{0}\right) \hat{\Psi}_{H}^{\dagger}\left(x^{\prime} t^{\prime}\right) \hat{\Psi}_{H}(x t)\right]}{\operatorname{Tr}\left[\hat{U}\left(t_{0}-i \beta, t_{0}\right)\right]},
$$

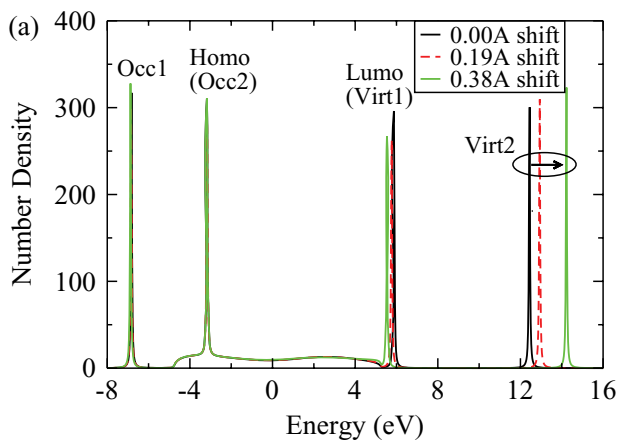

where $\hat{U}\left(t_{0}-i \beta, t_{0}\right)$ is the grand canonical density operator,

$$
\hat{U}\left(t_{0}-i \beta, t_{0}\right)=e^{-\beta\left(\hat{H}_{0}-\mu \hat{N}\right)}
$$

and can be viewed as a propagator of the time-independent Hamiltonian, $\hat{H}_{0}=\int h\left(x t_{0}\right) \hat{\Psi}_{H}^{\dagger}\left(x t_{0}\right) \hat{\Psi}_{H}\left(x t_{0}\right) d x$, offset by the electron number operator, $\hat{N}=\int \hat{\Psi}_{H}^{\dagger}\left(x t_{0}\right) \hat{\Psi}_{H}\left(x t_{0}\right) d x$, at equilibrium conditions designated by a given initial chemical potential $\mu$ and temperature $\beta \equiv\left(k_{B} T\right)^{-1}$. This propagator then acts over the complex time interval $\left[t_{0}, t_{0}-i \beta\right]$. The traces in Eq. (5) are taken over states of all possible energies and electron numbers. The field operator $\hat{\Psi}_{H}^{\dagger}\left(x^{\prime} t^{\prime}\right)$ $\left(\hat{\Psi}_{H}(x t)\right)$ is a sum of single electron creation (annihilation) operators weighted by corresponding single electron states in the Heisenberg picture. For example,

$$
\hat{\Psi}_{H}(x t)=\hat{U}\left(t_{\circ}, t\right)\left[\sum_{j} \psi_{j}(x) \hat{a}_{j}\right] \hat{U}\left(t, t_{\circ}\right),
$$

where $\hat{U}\left(t, t^{\prime}\right)$ propagates the full time-dependent Hamiltonian, $\hat{H}_{0}+\hat{V}(t)-\mu \hat{N}=\int h(x t) \hat{\Psi}_{H}^{\dagger}\left(x t_{0}\right) \hat{\Psi}_{H}\left(x t_{0}\right) d x$, over the real time interval $\left[t^{\prime}, t\right]$. Note that we have chosen to absorb $\mu$ into $h(x t)$. This convention will be followed from this point forward. The numerator in Eq. (5) can be viewed as containing a sequence of propagators and field operators. This sequence defines the Keldysh contour (Fig. 5).

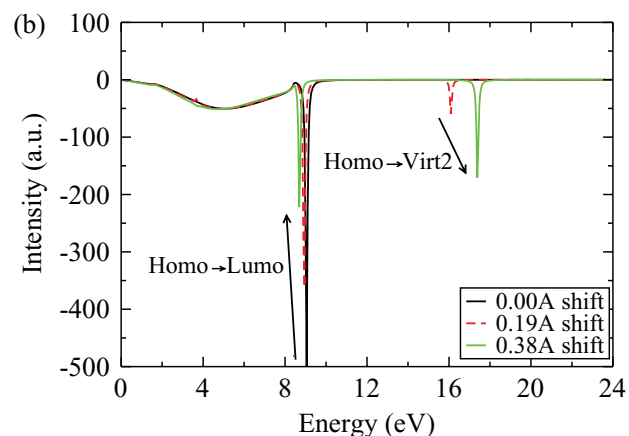

FIG. 4. (a) The electronic DOS under carbon shifting toward an electrode at equilibrium. (b) The electronic spectra under the carbon shifting. The Homo-Virt2 transition is enabled by the symmetry breaking. 


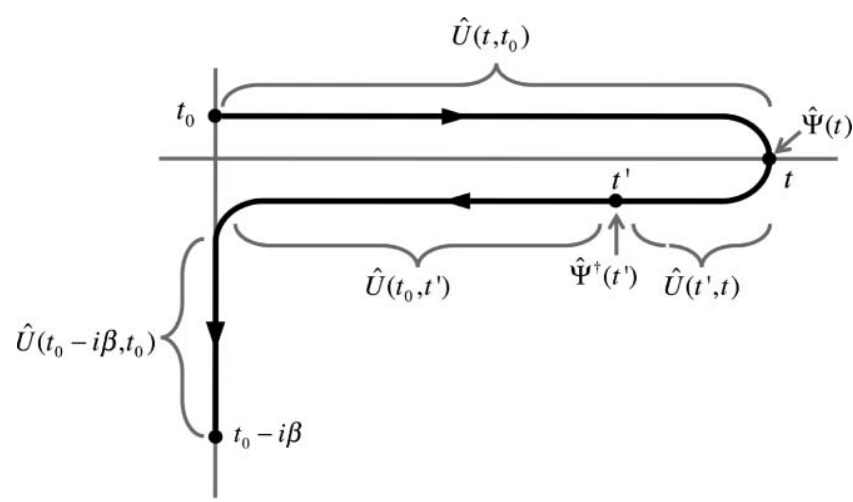

FIG. 5. The Keldysh time contour includes three branches defined in the complex plane: forward and backward real time and, extended into the imaginary axis at $t_{0}$, the thermal lifetime. We vertically shift the forward and backward branches along the real axis for purposes of illustration.

In the absence of explicit electron correlation and exchange terms, the lesser GF solves the e.o.m.,

$$
\begin{aligned}
& {\left[i \frac{\partial}{\partial t}-h(x t)\right] G^{<}\left(x t, x^{\prime} t^{\prime}\right)=0,} \\
& {\left[G^{<}\left(x t, x^{\prime} t^{\prime}\right)\right]^{*}=-G^{<}\left(x^{\prime} t^{\prime}, x t\right),}
\end{aligned}
$$

where $h(x t)$ is the one-body Hamiltonian describing the system. Here we use the variable $x$ to represent a vector of the spatial coordinates and use atomic units, where $\hbar \equiv 1$. We express operators in a localized diadic basis representation, where, for example, the propagated $G^{<}$becomes

$$
G^{<}\left(x t, x^{\prime} t^{\prime}\right)=\sum_{i, j} G_{i, j}^{<}\left(t, t^{\prime}\right) \phi_{i}(x) \phi_{j}\left(x^{\prime}\right),
$$

and $h_{i, j}(t)=\int d x \phi_{i}(x) h(x t) \phi_{j}(x)$. Note that a localized nonorthogonal basis set, such as the Gaussian basis sets typically used in electronic structure theory, can be rotated to a localized orthogonal basis set. Therefore, without loss of generality, Eqs. (8) and (9) become

$$
\begin{aligned}
& {\left[i \mathbf{1} \frac{\partial}{\partial t}-\mathbf{h}(t)\right] \mathbf{G}^{<}\left(t, t^{\prime}\right)=0,} \\
& {\left[\mathbf{G}^{<}\left(t, t^{\prime}\right)\right]^{\dagger}=-\mathbf{G}^{<}\left(t^{\prime}, t\right) .}
\end{aligned}
$$

The complete one-body Hamiltonian takes the following form:

$$
\mathbf{h}(\mathbf{t})=\mathbf{h}_{\mathbf{0}}+\mathbf{v}(t)
$$

where $\mathbf{h}_{\mathbf{0}}$ is defined by the model and the TD component $\mathbf{v}(t)$ is the probing dipole perturbation also described above. We combine the two equations and rewrite in terms of the time variables: $\bar{t} \equiv\left(t+t^{\prime}\right) / 2$ and $\Delta t \equiv t-t^{\prime}$ to obtain

$$
\begin{aligned}
& \frac{\partial}{\partial \bar{t}} \mathbf{G}^{<}(\bar{t}, \Delta t) \\
& \quad=i\left[\mathbf{G}^{<}(\bar{t}, \Delta t) \mathbf{h}\left(\bar{t}-\frac{\Delta t}{2}\right)-\mathbf{h}\left(\bar{t}+\frac{\Delta t}{2}\right) \mathbf{G}^{<}(\bar{t}, \Delta t)\right] .
\end{aligned}
$$

The two-time variable GF is the correlation function of an electronic system coupled to electron reservoirs as expressed on the Keldysh contour. ${ }^{38,39}$ The dynamical electronic density $(\rho(E, t)$ in Eq. (3)) can be extracted from this GF by Fourier transforming from the $\Delta t$ domain to the frequency $(\bar{\omega})$ or en$\operatorname{ergy}(E=\hbar \bar{\omega})$ domain, ${ }^{33}$

$$
\rho(E, \bar{t})=-i \int_{-\infty}^{\infty} d(\Delta t) e^{i E \Delta t} \mathbf{G}^{<}(\bar{t}, \Delta t) .
$$

The first transformation over $\Delta t$ results directly with the desired evolving electronic distribution. The bulk projection is based on using the electrode's self-energy expressed by $\Sigma$ 's. The corresponding $G^{<}$electronic Kadanoff-Baym (K-B) e.o.m.s ${ }^{38,39}$ are written as

$$
\begin{aligned}
i \frac{\partial}{\partial \bar{t}} \Delta \mathbf{G}^{<}(\bar{t}, \bar{\omega})= & {\left[\mathbf{h}_{\mathbf{0}}+\mathbf{v}_{\mathbf{0}}, \Delta \mathbf{G}^{<}(\bar{t}, \bar{\omega})\right]+\int d \omega^{\prime}\left[\mathbf{v}\left(\bar{t}, \omega^{\prime}\right) \mathbf{G}^{<}\left(\bar{t}, \bar{\omega}-\omega^{\prime}\right)-\mathbf{G}^{<}\left(\bar{t}, \bar{\omega}+\omega^{\prime}\right) \mathbf{v}\left(\bar{t}, \omega^{\prime}\right)\right] } \\
& +\int_{-\infty}^{\infty} d t^{\prime}\left[\Sigma^{\mathbf{R}}\left(\bar{t}-t^{\prime}\right) \Delta \mathbf{G}^{<}\left(t^{\prime}, \bar{\omega}\right) e^{-i \mathbf{h}_{\mathbf{0}}\left(\bar{t}-t^{\prime}\right)}-e^{i \mathbf{h}_{\mathbf{0}}\left(\bar{t}-t^{\prime}\right)} \Delta \mathbf{G}^{<}\left(t^{\prime}, \bar{\omega}\right) \Sigma^{\mathbf{A}}\left(t^{\prime}-\bar{t}\right)\right] .
\end{aligned}
$$

The full derivation of Eq. (16) is provided in Ref. 33. The self-energy $(\Sigma)$ terms above introduce the effects of the electrodes by projecting the GFs for the time-independent electrode Hamiltonian, $\mathbf{g}^{\mathbf{R}}{ }_{i}(t)$, onto the device,

$$
\Sigma^{\mathbf{R}}(t)=\sum_{i} \mathbf{h}_{0 i} \mathbf{g}^{\mathbf{R}}{ }_{i}(t) \mathbf{h}_{0 i}^{\dagger} .
$$

Here $\mathbf{h}_{0 i}$ is the electrode device hopping term for the $i$ th electrode in the time-independent Hamiltonian. Several methods exist for calculating $\mathbf{g}^{\mathbf{R}}$. We use a decimation tech- nique that, in effect, iteratively renormalizes the tight-binding Hamiltonian for a periodic semi-infinite electrode. ${ }^{40,41}$

The $G^{<}$of the bridging system written in the mixed time-frequency representation can be separated into timedependent and time-independent components:

$$
\mathbf{G}^{<}(\bar{t}, \bar{\omega})=\mathbf{G}_{v_{0}}^{0,<}(\bar{\omega})+\Delta \mathbf{G}^{<}(\bar{t}, \bar{\omega}) .
$$

In this picture, the $\Delta \mathbf{G}^{<}(\bar{t}, \bar{\omega})$ is defined to be the difference between the total lesser GF, $\mathbf{G}^{<}(\bar{t}, \bar{\omega})$, and the steady state lesser GF, $\mathbf{G}_{v_{0}}^{0,<}(\bar{\omega})$, under the effect of a constant bias $\left(\mathbf{v}_{0}\right)$. At steady state, the electronic density reduces to an energy 
distribution that results from coupling to electrodes. The electronic density distribution can be extracted from the lesser GF, where $G^{<}\left(t_{1}, t_{2}\right) \rightarrow G^{<}\left(t_{1}-t_{2}\right)$, and therefore, $\rho(E, \bar{t})$ $\rightarrow \rho(E)$. This form is appropriate for a PT treatment.

We re-express the GFs using Fourier transforms and solve for the response of the system to a TD perturbation $v(t)$ using PT. In this approach, the excitation spectrum of the biased system is obtained from the first order electronic response to the instantaneously impulsive perturbing potential [see Eq. (4)]. As reference for the PT treatment, we use the simplified e.o.m. under steady biasing conditions $\left(G_{v_{0}}^{0,<}(\bar{\omega})\right)$. The energy distribution $\mathbf{v}(\bar{t}, \omega)$ of the TD perturbation acting on the electrode-coupled system, $\mathbf{v}(t)$, is given in terms of the perturbation's Fourier transform $\tilde{\mathbf{v}}(2 \bar{\omega})$,

$$
\mathbf{v}(\bar{t}, \bar{\omega})=\frac{1}{\pi} e^{-i 2 \bar{\omega} \bar{t}} \int_{-\infty}^{\infty} d t e^{i(2 \bar{\omega}) t} \mathbf{v}(t)=\frac{1}{\pi} e^{-i 2 \bar{\omega} \bar{t}} \tilde{\mathbf{v}}(2 \bar{\omega}) .
$$

In calculating $\mathbf{G}_{v_{0}}^{0,<}(\bar{\omega})$, we use the relationship between $\mathbf{G}^{<}(\bar{\omega})$ and the retarded GF, $\mathbf{G}^{R}(\bar{\omega})$, which entails calculating the Fermi matrix. ${ }^{33}$

In the full frequency representation, where $\mathrm{G}^{<}(\Delta \omega, \bar{\omega})$ $\equiv \int_{-\infty}^{\infty} d \bar{t} e^{i \Delta \omega \bar{t}} \mathrm{G}^{<}(\bar{t}, \bar{\omega})$, the application of the TD-PT treatment becomes more effective than in the mixed representation. In the PT expansion expressed in the frequency domain, the bulk self-energies are written exactly, for example, without using the wide band approximation. The e.o.m. takes the following form: ${ }^{42}$

$$
\begin{aligned}
\sum_{k, l} & \mathcal{H}_{i j k l}(\Delta \omega) \Delta G_{k l}^{<}(\Delta \omega, \bar{\omega})=B_{i j}^{(1)}(\Delta \omega, \bar{\omega})_{i j} \\
+ & \frac{1}{\pi} \int d \omega^{\prime}\left[\tilde{\mathbf{v}}\left(2 \omega^{\prime}\right) \Delta \mathbf{G}^{<}\left(\Delta \omega-2 \omega^{\prime}, \bar{\omega}-\omega^{\prime}\right)\right. \\
& \left.-\Delta \mathbf{G}^{<}\left(\Delta \omega-2 \omega^{\prime}, \bar{\omega}+\omega^{\prime}\right) \tilde{\mathbf{v}}\left(2 \omega^{\prime}\right)\right]_{i j},
\end{aligned}
$$

where

$$
\begin{aligned}
\mathcal{H}_{i j k l}(\Delta \omega) \equiv & \left(\Delta \omega+i \eta-\Delta \epsilon_{i j}\right) \delta_{i k} \delta_{j l} \\
& -\Gamma_{i j k l}(\Delta \omega)-\left(v_{0 i k} \delta_{l j}-v_{0 l j} \delta_{i k}\right) .
\end{aligned}
$$

Here

$$
\begin{aligned}
& \mathbf{B}^{(\mathbf{1})}(\Delta \omega, \bar{\omega}) \\
& \quad \equiv\left[\tilde{\mathbf{v}}(\Delta \omega) \mathbf{G}_{v_{0}}^{0,<}(\bar{\omega}-\Delta \omega / 2)-\mathbf{G}_{v_{0}}^{0,<}(\bar{\omega}+\Delta \omega / 2) \tilde{\mathbf{v}}(\Delta \omega)\right],
\end{aligned}
$$

and $\Gamma_{i j k l}(\Delta \omega)$ is the broadening function due to coupling to the electrodes that is generalized to include dynamical effects (memory kernel) by defining

$$
\begin{aligned}
\Gamma_{i j k l}(\Delta \omega) & \equiv \int d t e^{i \Delta \omega t}\left[\Sigma_{i k}^{R}(t) e^{i \epsilon_{j} t} \delta_{l j}-\Sigma_{l j}^{A}(-t) e^{-i \epsilon_{i} t} \delta_{i k}\right] \\
& =\Sigma_{i k}^{R}\left(\epsilon_{j}+\Delta \omega\right) \delta_{l j}-\Sigma_{l j}^{A}\left(\epsilon_{i}-\Delta \omega\right) \delta_{i k} .
\end{aligned}
$$

In the above equation $\Delta \epsilon_{i j} \equiv \epsilon_{i}-\epsilon_{j}$ is the difference between the $i$-th and $j$-th eigenvalues $\left(\epsilon_{i}, \epsilon_{j}\right)$ of $h_{0}$. The bias $\left(\mathbf{v}_{\mathbf{0}}\right)$ effect is entered in the expansion treatment but also in defining the $\mathcal{H}$ superoperator. The implemented formalism includes a broadening factor $(\eta)$ in defining the tensor $\mathcal{H}$ [see Eq. (21)].
In Eq. (20), which can formally be expanded to arbitrary order in the perturbation, we express the TD electronic density in terms of the evolving occupations of the projected junction states. ${ }^{42}$ The band structure due to the electrode-coupling is included directly in this expansion through the energy distribution variable $\bar{\omega}$. We note that Eq. (20) involves a tensor of rank 4 that is contracted with a matrix (tensor of rank 2).

Here, we study the electronic spectrum that can be obtained from the linear response of the electronic density as discussed above. The convolution integral [second term in the RHS of Eq. (20)] is dropped in the first order expansion. Using tetradic notation, the tensor $\mathcal{H}_{i j k l}$ of rank 4 with $n$ dimensionality in each index is re-expressed as a matrix with elements $\mathrm{H}_{n i+j, n k+l}$ and $n^{2}$ dimensionality in each index, likewise a matrix becomes a vector. The first order expansion of Eq. (20) then becomes

$$
\left.\left.\mathrm{H}(\Delta \omega)\left|\Delta G^{<}(\Delta \omega, \bar{\omega})\right\rangle\right\rangle=\left|B^{(1)}(\Delta \omega, \bar{\omega})\right\rangle\right\rangle,
$$

where $\left.\mathrm{H}_{n i+j, n k+l}(\Delta \omega) \equiv \mathcal{H}_{i j k l}(\Delta \omega), \quad\left|\Delta G^{<}(\Delta \omega, \bar{\omega})\right\rangle\right\rangle_{n i+j}$ $\equiv \Delta G_{i j}^{<}(\Delta \omega, \bar{\omega})$ and $\left.\left|B^{(1)}(\Delta \omega, \bar{\omega})\right\rangle\right\rangle_{n i+j} \equiv B^{(1)}{ }_{i j}(\Delta \omega, \bar{\omega})$.

As noted above, we apply an instantaneously impulsive potential in the dipole approximation to calculate the spectral response,

$$
\mathbf{v}(t)=\mathbf{v}_{a} \delta(t)=\mathcal{E}_{0} \delta(t) \mathbf{D} .
$$

where $D_{i j}=\int d x \phi_{i}(x) \hat{D} \phi_{j}(x)$ and $\hat{D}=e \hat{x}$ is the dipole operator. In the frequency domain, such a pulse is independent of frequency,

$$
\tilde{\mathbf{v}}(\Delta \omega)=\mathbf{v}_{a}=\mathcal{E}_{0} \mathbf{D}
$$

\section{RESULTS}

We first study the electrode-coupling effect (broadening of energy states) on the electronic spectrum. The electronic spectrum of the coupled system is provided in Fig. 3(b). We focus on the broadening effect, which can be tuned by the fundamental broadening factor in calculating the retarded GF, $\mathbf{G}^{R}(\bar{\omega})$, of the electrode-coupled system. The broadening of the electronic DOS leads to broadening of the main spectral peak related to the transition between the highest occupied molecular orbital (HOMO) and lowest unoccupied molecular orbital (LUMO). An additional widely broadened peak is observed at lower spectral frequencies and is associated with the band of states in the DOS located energetically between the HOMO and LUMO peaks.

We also study the combined effect of bias and molecular scale motion on the spectrum. The carbon atoms are shifted by increments of $0.19 \AA$ toward either electrode, where the resulting molecular electronic DOS is provided in Fig. 4. The two occupied states respond only slightly to the carbon shift. More substantial changes are observed for the virtual state energies, where a larger positive shift of the higher virtual state is noted. The corresponding spectra are provided in Fig. 4(b). Upon the symmetry breaking shift, the symmetry forbidden HOMO-Virt2 transition becomes allowed (Virt2 denotes the higher unoccupied state, which is the LUMO+1). The two spectral peaks are shifted to higher and lower values 

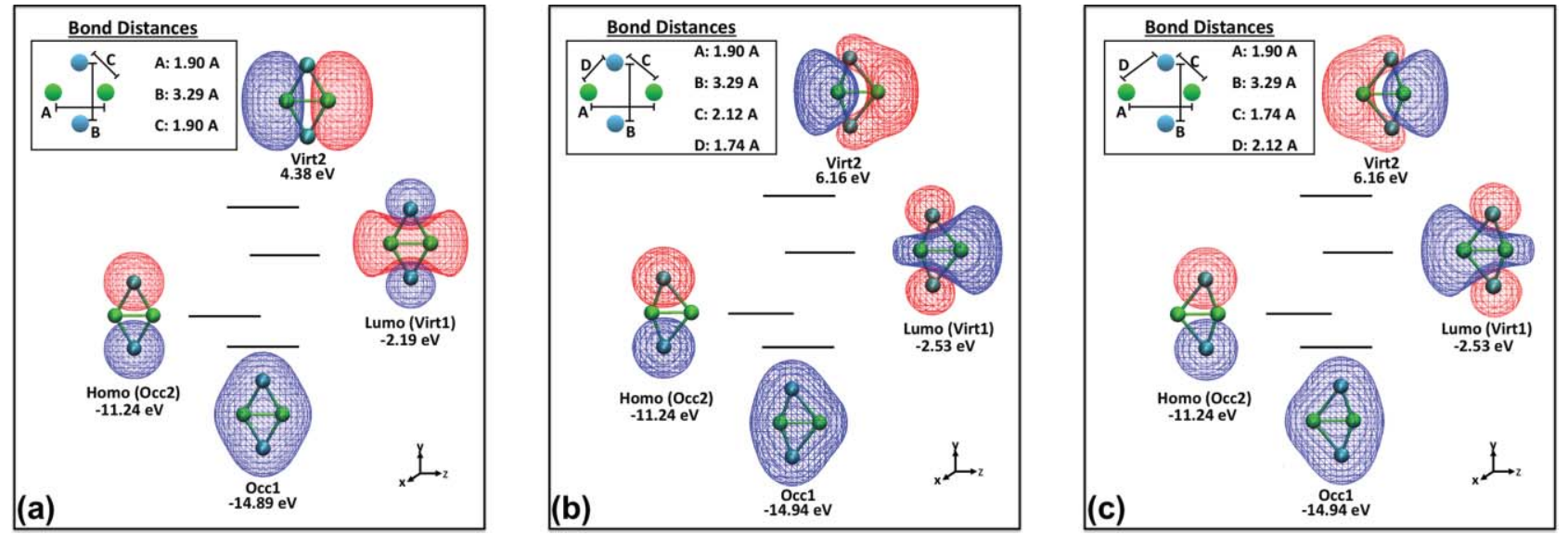

FIG. 6. Molecular orbital diagrams for (a) nonshifted case and shift of $0.38 \AA$, (b) toward source electrode, and (c) toward drain electrode. Relevant bond lengths and energies are included.

in energy, corresponding to the changes in the virtual state energy.

It is important to comment on the HOMO-LUMO transition, where symmetry-induced disabling effects are noted. The molecular orbital picture for the core region at equilibrium (no bias applied) is provided in Fig. 6. The orbital energies, corresponding to Fermi energy of $-8.067 \mathrm{eV}$, are provided along with the orbital assignment. The even/odd symmetry of the original orbitals is broken when the carbons are shifted toward the electrode. Clearly, at equilibrium, shifting the carbon atoms toward either electrode has the same effect on the electronic DOS and therefore on the spectrum. Next we consider the electronic spectrum under applied bias.

Potential bias is applied to the geometrically symmetric system in increments of $2 \mathrm{~V}$. The resulting flux populates and depopulates the virtual and occupied orbitals, respectively. Specifically, the electron flux partially occupies the LUMO and partially depletes the HOMO, affecting the spectrum fundamentally. First, the spectral cross section of the corresponding allowed excitation in the unbiased system is reduced. As the bias is increased, the HOMO-LUMO transition is disabled, as illustrated in Fig. 7. The dynamical occupation of the orbitals also affects the spectrum by enabling new transitions that are otherwise absent for the unbiased system. Upon

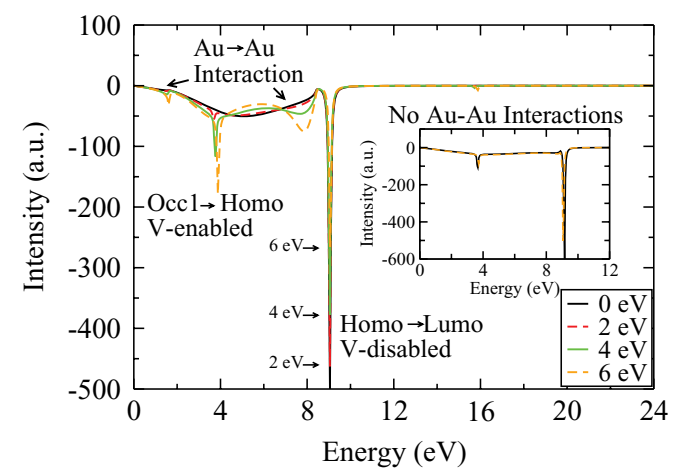

FIG. 7. Spectral dependence on increasing potential bias for geometrically symmetric junction. (Inset) Bias dependence for small device region indicates cause of $\mathrm{Au}-\mathrm{Au}$ interaction. bias induced flux, the Occ1-HOMO transition becomes enabled and increases with increasing applied bias (Occ1 denotes the lower occupied, or HOMO-1 state).

On a side note, we confirm the assignment of the biasenabled peak to the Occ1-HOMO excitations. The same peak appears in the spectrum of a model device that includes only one gold atom on each side, as shown under biases ranging from 0 to $6 \mathrm{~V}$ (insert to Fig. 7). This model, while insufficient for converging the representation of electrode-coupling, confirms that the additional peaks in the low energy regime are assigned only to intragold wire transitions (Fig. 7).

We now consider the application of bias potential to the shifted (symmetry broken) carbon systems. Figures 8(a) and 8 (b) show the spectra when the carbons are shifted toward the source and drain electrode, respectively. We focus on the effect of potential bias on the HOMO-LUMO transition for different geometric shifts. The bias-disabling of this transition is enhanced when the carbons are shifted toward the source. The opposite effect occurs when the symmetry breaking involves a shift toward the drain. In this case, the bias-disabling is diminished and the spectrum features a small increase of the bias-induced peak as well as a shift to higher excitation energies.

To understand the reason for this difference, we reconsider the electronic orbitals illustrated in Fig. 6 in relation to applied bias. For example, we focus on the LUMO where the symmetry breaking results in a shift of the projected density to the more distant gold atom. The symmetry-breaking-induced polarization is enhanced, therefore, by flux-induced polarization when the molecule is shifted toward the source. On the other hand, the flux negates the symmetry-breaking-induced polarization of the LUMO when the carbons are shifted toward the drain. Therefore, the bias-disabling effect on the HOMO-LUMO spectral peak is enhanced when the geometric shift is oriented toward the source and is decreased when the shift is oriented toward the drain.

The symmetry breaking is more subtle in the case of the occupied orbital transitions. Unlike the LUMO, the symmetry breaking shifts the projected density toward the closer gold atom. This slight symmetry breaking of the occupied 

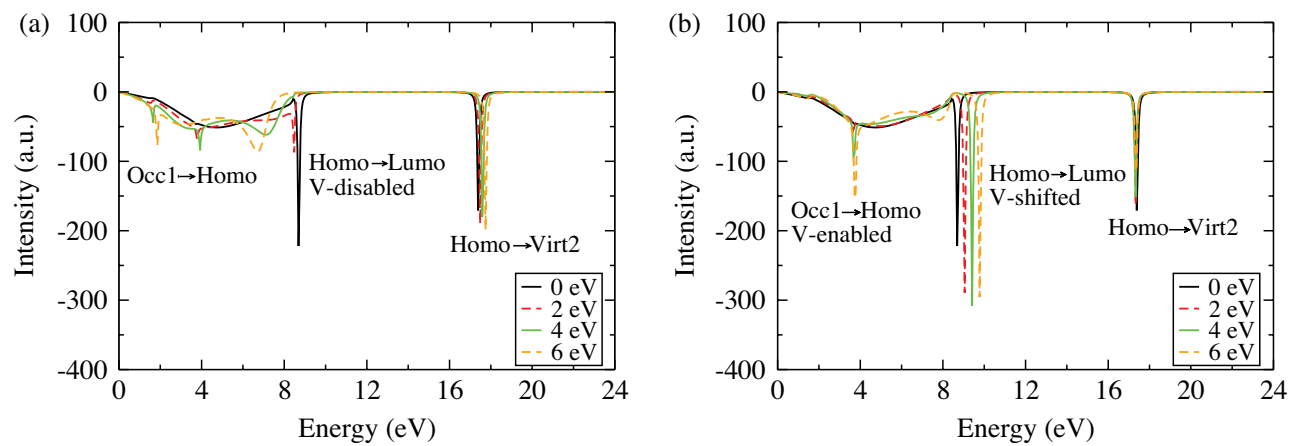

FIG. 8. The spectral dependence on increasing bias under carbon shift toward (a) source electrode and (b) drain electrode.

orbitals is reversed by bias-induced flux upon shifting the carbons toward the source and is enhanced when the carbons are shifted toward the drain. Enabling of the Occ1-HOMO transition requires that the HOMO level becomes (at least partially) unoccupied. We find that increasing bias enhances the biasenabled Occ1-HOMO peak for the geometric shift toward the drain. This peak appears and then is reduced with further increase in bias for the shift toward the source. The effect on the Occ1-HOMO transition can be attributed to the transmission of each orbital due to electrode-coupling. When coupled to the drain, the transport through the HOMO is decreased, allowing the Occ1-HOMO transition to occur. When coupled to the source, the transport through the HOMO increases as the bias increases, causing the orbital to remain occupied and the Occ1-HOMO transition to become disabled.

\section{CONCLUSIONS}

The effects of electrode-coupling and bias on the electronic spectrum of a simple molecular model system are analyzed. Fundamentally, electrode-coupling leads to broadened electronic spectral peaks. Biasing conditions can lead to further substantial changes in the electronic spectrum. Under bias, spectral peaks can be enabled by the dynamical occupation and depletion of levels due to the electron flux. The dynamical occupation, therefore, reduces spectral peaks that are present at equilibrium conditions but can also enable additional transitions, for example, those between equilibrium occupied levels. Finally, symmetry breaking molecular motion fundamentally affects the spectrum by enabling symmetry forbidden transitions. The bias effect on the spectrum can be negated or enhanced by this symmetry breaking of molecular orbitals. Indeed, we find, for example, that the biasenabled transition between two occupied states is maintained in the drain-coupled case, since the electronic population of the higher occupied level depletes more effectively than in the source-coupled case.

\section{ACKNOWLEDGMENTS}

The work of Barray D. Dunietz was supported as part of the Center for Solar and Thermal Energy Conversion, an Energy Frontier Research Center funded by the U.S.
Department of Energy, Office of Science, Office of Basic Energy Sciences under Award Number DE-SC0000957. Heidi Phillips is thankful for support from the University of Michigan Rackham Graduate School Summer Institute and the University of Michigan Rackham Merit Fellowship.

${ }^{1}$ L. A. Bumm, J. J. Arnold, M. T. Cygan, T. D. Dunbar, T. P. Burgin, L. I. Jones, D. L. Allara, J. M. Tour, and P. S. Weiss, Science 271, 1705 (1996).

${ }^{2}$ M. A. Reed, C. Zhou, C. J. Muller, T. P. Burgin, and J. M. Tour, Science 278, 252 (1997).

${ }^{3}$ R. E. Holmlin, R. Haag, M. L. Chabinyc, R. F. Ismagilov, A. E. Cohen, A. Terfort, M. A. Rampi, and G. M. Whitesides, J. Am. Chem. Soc. 123, 5075 (2001).

${ }^{4}$ X. D. Cui, A. Primak, X. Zarate, J. Tomfohr, O. F. Sankey, A. L. Moore, T. A. Moore, D. Gust, G. Harris, and S. M. Lindsay, Science 294, 571 (2001).

5 J. Reichert, R. Ochs, D. Beckmann, H. B. Weber, M. Mayor, and H. V. Löhneysen, Phys. Rev. Lett. 88, 176804 (2002).

${ }^{6}$ J. G. Kushmerick, D. B. Holt, S. K. Pollack, M. A. Ratner, J. C. Yang, T. L. Schull, J. Naciri, M. H. Moore, and R. Shashidhar, J. Am. Chem. Soc. 124, 10654 (2002).

${ }^{7}$ J. K. N. Mbindyo, T. E. Mallouk, J. B. Mattzela, I. Kratochvilova, B. Razavi, T. N. Jackson, and T. S. Mayer, J. Am. Chem. Soc. 124, 4020 (2002).

${ }^{8}$ B. Q. Xu and N. Tao, Science 301, 1221 (2003).

${ }^{9}$ B. Q. Xu, X. Y. Xiao, X. Yang, L. Zang, and N. J. Tao, J. Am. Chem. Soc. 127, 2386 (2005).

${ }^{10}$ X. Guo, J. P. Small, J. E. Klare, Y. Wang, M. S. Purewal, I. W. Tam, B. H. Hong, R. Caldwell, L. Huang, S. O’Brien, J. Yan, R. Breslow, S. J. Wind, J. Hone, P. Kim, and C. Nuckolls, Science 311, 356 (2006).

${ }^{11}$ J. Y. Tang, Y. L. Wang, J. E. Klare, G. S. Tulevski, S. J. Wind, and C. Nuckolls, Angew. Chem., Int. Ed. 46, 3892 (2007).

${ }^{12}$ X. Chen, Y.-M. Jeon, J.-W. Jang, L. Qin, F. Huo, W. Wei, and C. A. Mirkin, J. Am. Chem. Soc. 130, 8166 (2008).

${ }^{13}$ H. Basch, R. Cohen, and M. A. Ratner, Nano Lett. 5, 1668 (2005).

${ }^{14}$ T. Perrine and B. D. Dunietz, Phys. Rev. B 75, 195319 (2007).

${ }^{15}$ W. Ho, J. Chem. Phys. 117, 11033 (2002).

${ }^{16}$ W. Wang, T. Lee, I. Kretzschmar, and M. A. Reed, Nano Lett. 4, 643 (2004).

${ }^{17}$ Y. Selzer, L. Cai, M. A. Cabassi, Y. Yao, J. M. Tour, T. S. Mayer, and D. L. Allara, Nano Lett. 5, 61 (2005).

${ }^{18}$ M. Galperin, M. Ratner, and A. Nitzan, J. Chem. Phys. 121, 11965 (2004).

${ }^{19}$ N. Sergueev, D. Roubtsov, and H. Guo, Phys. Rev. Lett. 95, 146803 (2005)

${ }^{20}$ R. Hartle, C. Benesch, and M. Thoss, Phys. Rev. Lett. 102, 146801 (2009).

${ }^{21}$ D. A. Ryndyk, M. Hartung, and G. Cuniberti, Phys. Rev. B 73, 045420 (2006).

${ }^{22}$ M. Galperin, M. A. Ratner, and A. Nitzan, J. Phys.: Condens. Matter 19, 103201 (2007).

${ }^{23}$ A. M. Nowak and R. L. McCreery, J. Am. Chem. Soc. 126, 16621 (2004).

${ }^{24}$ A. M. Nowak and R. L. McCreery, Anal. Chem. 76, 1089 (2004).

${ }^{25}$ A. P. Bonifas and R. L. McCreery, Chem. Mater. 20, 3849 (2008). 
${ }^{26}$ D. R. Ward, N. J. Halas, J. W. Ciszek, J. M. Tour, Y. Wu, P. Nordlander, and D. Natelson, Nano Lett. 8, 919 (2008).

${ }^{27}$ D. R. Ward, G. D. Scott, Z. K. Keane, N. J. Halas, and D. Natelson, J. Phys.: Condens. Matter 20, 374118 (2008).

${ }^{28}$ N. Bushong, N. Sai, and M. D. Ventra, Nano Lett. 5, 2569 (2005).

${ }^{29}$ C.-L. Cheng, J. S. Evans, and T. Va. Voorhis, Phys. Rev. B 74, 155112 (2006).

${ }^{30}$ J. Maciejko, J. Wang, and H. Guo, Phys. Rev. B 74, 085324 (2006).

${ }^{31}$ D. Hou, Y. He, X. Liu, J. Kang, J. Chen, and R. Han, Physica E 31, 191 (2006).

${ }^{32}$ N. Sai, N. Bushong, R. Hatcher, and M. D. Ventra, Phys. Rev. B 75, 115410 (2007).

${ }^{33}$ A. Prociuk and B. D. Dunietz, Phys. Rev. B 78, 165112 (2008).

${ }^{34}$ G. Stefanucci, S. Kurth, A. Rubio, and E. K.U. Gross, Phys. Rev. B 77, 075339 (2008)
${ }^{35}$ A. Prociuk and B. D. Dunietz;; "On the electronic spectra of a molecular bridge under non-equilibrium electric potential conditions," in $\mathrm{Ad}$ vances in the Theory of Atomic and Molecular Systems, Dynamics, Spectroscopy, Clusters, and Nanostructures, Progress in Theoretical Chemistry and Physics (Springer, Netherlands, 2009), Vol. 20, pp. 265-277.

${ }^{36}$ A. B. Pacheco and S. S. Iyengar, J. Chem. Phys. 133, 044105 (2010).

${ }^{37}$ A. Prociuk and B. D. Dunietz, Phys. Rev. B 82, 125449 (2010).

${ }^{38}$ L. P. Kadanoff and G. Baym, Quantum Statistical Mechanics (Benjamin and Cummings, New York, 1962)

${ }^{39}$ M. Bonitz, Quantum Kinetic Theory (Teubner, Stuttgart, 1998).

${ }^{40}$ M. P.L. Sancho, J. M.L. Sancho, and J. Rubio, J. Phys. F: Met. Phys. 14, 1205 (1984)

${ }^{41}$ M. P.L. Sancho, J. M. L. L. Sancho, and J. Rubio, J. Phys. F: Met. Phys. 15, 851 (1985).

${ }^{42}$ A. Prociuk, H. Phillips, and B. D. Dunietz, J. Phys.: Conf. Ser. 220, 012008 (2010). 